\title{
Classification of Cardiovascular Disease from ECG using Artificial Neural Network and Hidden Markov Model.
}

\author{
${ }^{1}$ Mr. Ankit Sanghavi, ${ }^{2}$ Prof. Sachin M. Bojewar \\ ${ }^{1} P G$ Scholar, Department of Computer Engineering Alamuri Ratnamala Institute of Engineering \& Technology \\ Mumbai \\ ${ }^{2}$ Asst. Professor, Department of Computer Engineering Vidyalankar Institute of Engineering \& Technology \\ Mumbai
}

\begin{abstract}
If future progression of the disease can be predicted earlier with proper change in medication patients treatment can be improved. Artificial neural network (ANN) is used as classifier with wavelet transform as the feature extraction for reducing data set of ECG. Hidden markov model (HMM) is used as predictor along with artificial neural network (ANN). ECG samples are collected for testing from MIT_BIH database. MATLAB 2010b is used as the simulation tool for modeling and testing. The result obtain with Artificial neural network (ANN) and Hidden markov model (HMM) combine are much efficient than that of ANN alone.
\end{abstract}

Index Terms: cardiovascular disease classification, ECG, Wavelet transforms, artificial neural networks (ANN), hidden markov model (HMM).

\section{Introduction}

ECG has a basic role in heart related problem detection because it consists of effective, simple, noninvasive, low-cost procedures for the diagnosis of cardiovascular disorders that have a high mortality rate and are very relevant for their impact on patient's daily life and large amount of money. Asechocardiography or tallium scintigraphy imaging techniques offer diagnostic evidence in some instances, particularly in reduction of myocardial perfusion, so its unique attributes reserve a major role to electrocardiography in the diagnosis of cardiovascular diseases.

Pathological alterations observable by Electrocardiography

can be divided into three main areas:

1) Arrhythmia which is known as cardiac rhythm disturbances

2) Cardiac ischemia which is dysfunction of myocardial blood perfusion.

3) Left ventricular hypertrophy which means chronic alteration of the mechanical structure of the heart.

These are the importance of these disturbances is reflected by the availability of only three types of independent databases for performance evaluation of the algorithms for ECG analysis. They consist of archives of several annotated signals representative of arrhythmia, ischemia, and chronic diseases for the cardiovascular diseases.

Moreover, the available equipment implement separately only one specific procedure at a time out of these areas of cardiac Pathology.

The literature in this topic reports several approaches to classification, including Bayesian [11] and heuristic approaches [12], expert systems [13], and Markov models [14]. In general, past approaches, according to published results, seem to suffer from common drawbacks that depend on high sensitivity to noise and unreliability in dealing with new or ambiguous patterns.

Artificial neural networks (ANN's) have often been proposed as tools for realizing classifiers that are able to deal even with nonlinear discrimination between classes and to accept incomplete or ambiguous input patterns. Recently, the Connectionist approach has also been applied to ECG analysiswith promising results [15]-[19].

Despite so many applications of ANN to ECG analysis, the lack of a complete, systematic, and exhaustive comparison with traditional methods currently implemented in ECG analyzers does not allow a more organized industrial planning of ANN applications as ECG analyzers.

The systems available today are not perfect to handle the uncertainty in proper way.

The algorithm for the problem is as given below:

1. Start

2. Data acquisition.

3. Filtering and normalization. 
4. Feature extractions.

5. PQRST wave point detection

6. HMM analysis.

7. ANN training/simulation.

8. Goto step 2 and repeat.

9. End.

\section{TOOLS}

MATLAB 2010B is used as the tool of choice for modelling, simulation and testing of ECG signals.MATLAB is used because the basic data type of the tool is matrices so representation and processing of ECG signals from MIT_BIH database is easy. The ECG data obtain is of .dat format.

\section{Result Analysis}

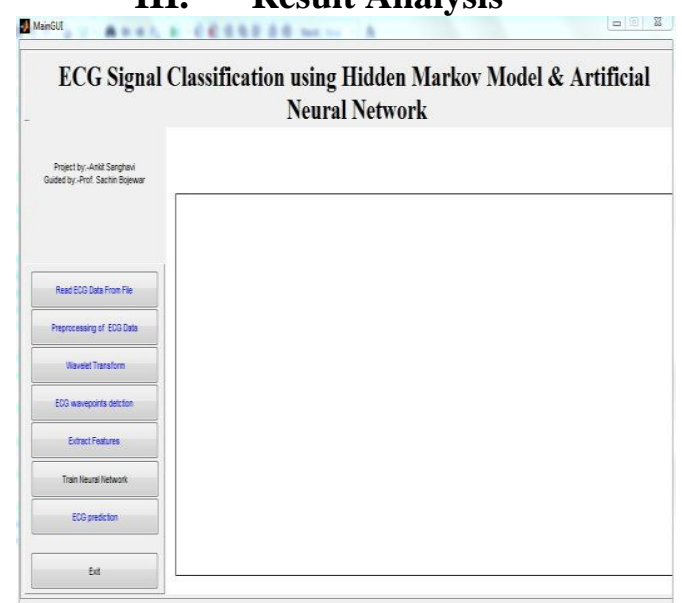

Fig.1.GUI for system implemented

Fig.1. shows the graphical user interface for the system implementation. GUIDE interface is used in matlab for designing the GUI.

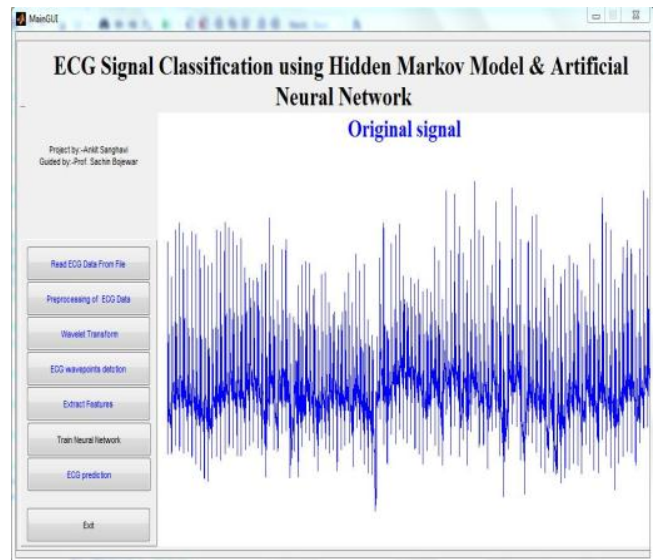

Fig.2.Original ECG signal

Fig.2 shows the ECG signal which is in .dat format available from MIT-BIH database. The original ECG signal is generated by placing two electrodes on patients chest near heart which measures the equivalent voltage againt the physical pumping of the heart which is then monitored on computer using the proprietary software like "capture". 


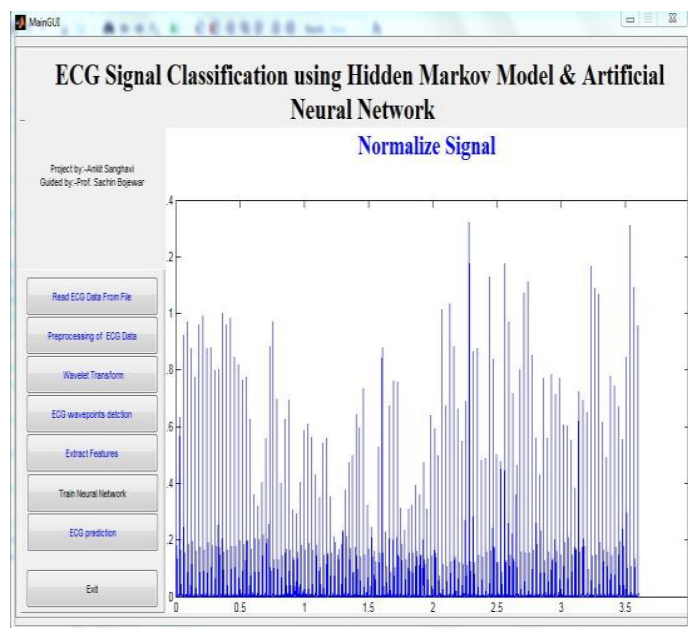

Fig.3.Filtered and normalized ECG signal

Fig.3 shows filtered output of ECG signal by using high pass notch filter which reduces the noise drastically associated with the ECG signal from MIT-BIH database.

The noise is generally thermal noise and white Gaussian noise associated with the ECG device. The noise removal is then followed by normalization of ECG signal.

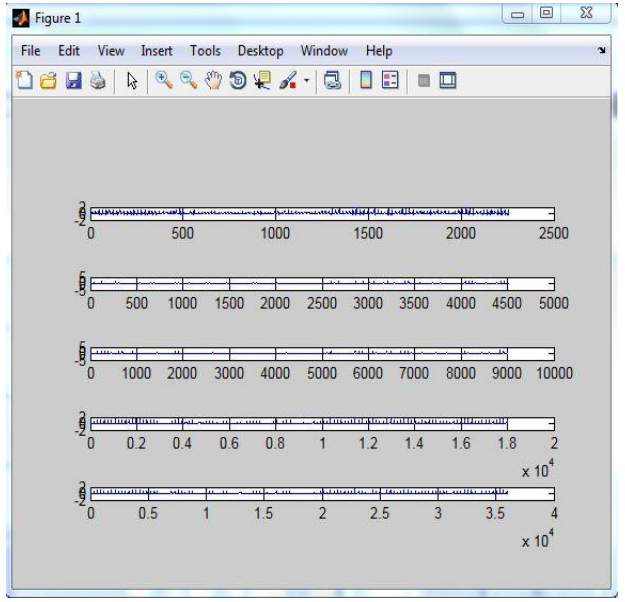

Fig.4.Wavelet coefficients

Wavelet transform is the widely used feature extraction technique for the problems of digital signal processing and digital image processing, which converts time domain signal into wavelet domain which is a kind of frequency representation. This wavelet decomposition is achieved using 'db6' scheme of Mallet's filtering.

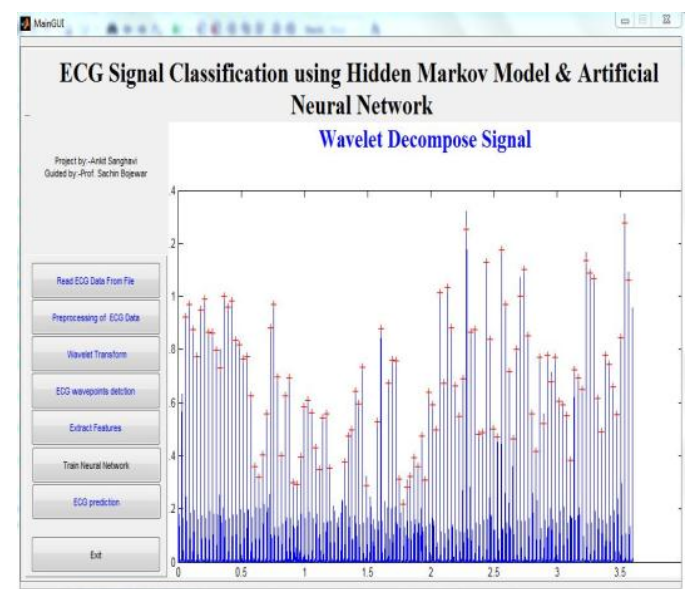

Fig.5.Wavelet decomposition 
Fig. 4 and Fig.5 give result for the wavelet decomposition and wavelet coefficients associated with the wavelet transform. Wavelet transform is used as feature extraction technique for the problems of digital signal processing and digital image processing, which converts time domain signal into wavelet domain which is a kind of frequency representation to reduce the ECG data points which is suitable as input for ANN.

This wavelet decomposition is achieved using 'db6' scheme of Mallet's filtering.

After performing wavelet transform PQRST wave points are detected using special algorithm. This step is essential for the modeling of hidden markov model along with the wavelet decompose signal. The red marks in above image show the starting and ending point of the ECG wave.

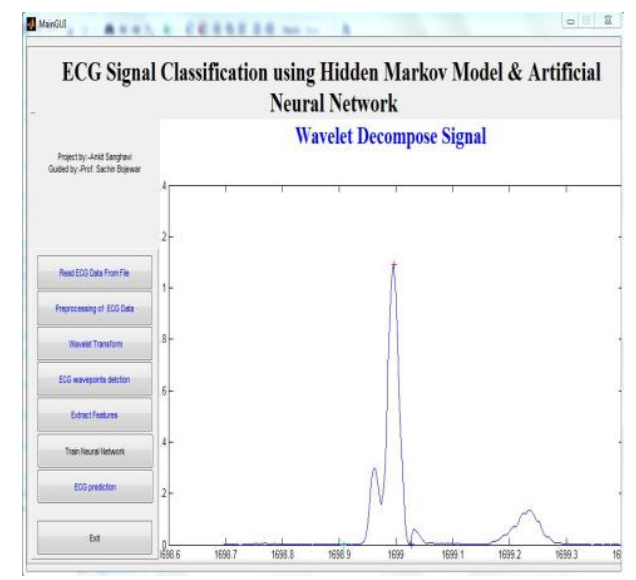

Fig.6.HMM output

The artificial neural network for training purpose requires the feature extracted values the capacity of the artificial neural network for the number of inputs are limited, above image shows the final feature extracted sequence which is to be assigned as input to the artificial neural network for the training. The efficiency of the artificial neural network is solely depends upon the feature extracted characteristics.

Fig. 6 shows the HMM model output we have implemented. This model implementation is as below: Let us generate nex $=50$ vector-valued sequences of length $\mathrm{T}=50$; each vector has size $\mathrm{O}=2$.

$\mathrm{O}=2$

$\mathrm{T}=50$

nex $=50$;

data $=\operatorname{randn}(\mathrm{O}, \mathrm{T}, \mathrm{nex})$

Now let use fit a mixture of $\mathrm{M}=2$ Gaussians for each of the $\mathrm{Q}=2$ states using $\mathrm{K}$-means.

$\mathrm{M}=2$;

$\mathrm{Q}=2$;

left_right $=0$;

prior0 $=$ normalise $(\operatorname{rand}(\mathrm{Q}, 1))$;

transmat $0=$ mk_stochastic $(\operatorname{rand}(\mathrm{Q}, \mathrm{Q}))$;

$[\mathrm{mu} 0, \operatorname{Sigma} 0]=$ mixgauss_init $\left(\mathrm{Q}^{*} \mathrm{M}, \operatorname{reshape}\left(\right.\right.$ data,$\left[\mathrm{O} \mathrm{T}^{*}\right.$ nex $\left.]\right)$, cov_type $)$,

$\mathrm{mu} 0=\operatorname{reshape}(\mathrm{mu} 0,[\mathrm{O} \mathrm{Q} \mathrm{M}])$;

Sigma0 = reshape $(\operatorname{Sigma0},[\mathrm{O} O \mathrm{Q} \mathrm{M}])$;

$\operatorname{mixmat} 0=$ mk_stochastic $(\operatorname{rand}(\mathrm{Q}, \mathrm{M}))$;

Finally, let us improve these parameter estimates using EM.

[LL, prior1, transmat1, mu1, Sigma1, mixmat1] $=\ldots$

mhmm_em(data, prior0, transmat0, mu0, Sigma0, mixmat0, 'max_iter', 2);

This HMM is used to find the Gaussian for The wavelet decomposition of ECG signal. 


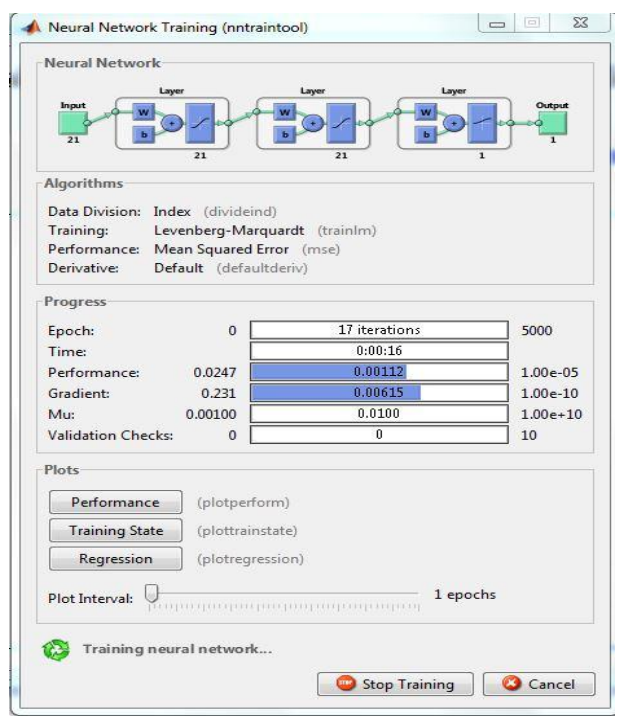

Fig.7.ANN training

The backpropogation feed forward neural network is used for the proposed system. The feature extracted data we obtained from the said process is assigned as an input to the neural network for the training, the Levenberg - Marquardt learning algorithm is used for the training of neural network. 30 Samples are used from the database as training data which is after simulation can be compared with other data sets.

Artificial neural network implementation is as given below:

$\mathrm{RC}=$ Regression and Curve fitting.

$\mathrm{CP}=$ Classification and Pattern Recognition

1. net1 =newff(minmax(p), [H O], TF*, BTF, BLF, PF ) \% RC and CP

=> 'purelin' tansig' 'logsig' output/No IPF, OPF, or DDF

2. a. net2 =newff(p, t, H, TF*, BTF, BLF, PF, IPF*, OPF*, DDF*) \% RC and CP $==>$ 'purelin' tansig' 'logsig'output/IPF, OPF and DDF defaults

b. net3 =newfit(p, t, H, TF, BTF, BLF, PF, IPF, OPF, DDF) \% RC (calls newff)

$==>$ same as newff with an added plotting function

c. net4 =newpr(p, t, H, TF*, BTF, BLF, PF, IPF, OPF, DDF) \% CP (calls newff)

$==>$ same as newff with 'tansig' output, 'trainlm' training and added CP plots

3. a. net5 =feedforwardnet $(\mathrm{H}, \mathrm{TF}) \% \mathrm{RC}$ and $\mathrm{CP}$

b. net $6=$ fitnet $(\mathrm{H}, \mathrm{TF}) \% \mathrm{RC}$ (calls feedforwardnet)

$==>$ same as feedfordnet with an added plot

c. net7 =patternnet ( H, TF ) \% CP (calls newff)

$==>$ same as newff with 'tansig' output, 'trainlm' training and added CP plots

1. Instead of trying to compare net 1 and net7, it is probably best to start with finding the differences among net1, net2 and net5.

2. Nets 2-7 have input and output normalization via MAPMINMAX defaults that results in changes in weight values. So, we don't think just assigning the same weights to the different nets will help.

3. The actual normalizations are probably performed in TRAIN. So it might be wise to override the IPF and OPF defaults and use TRAIN.

4. If TRAIN is used the DDF option should be overridden to make sure all of the data is used for training. 


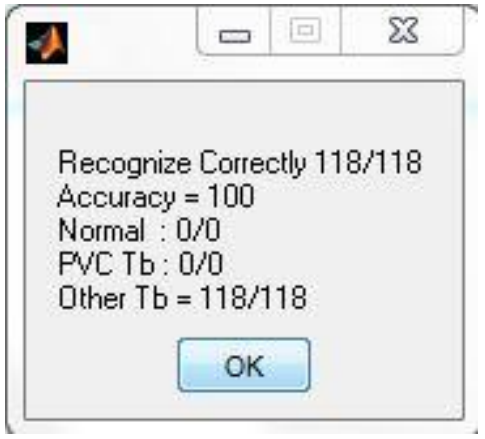

Fig.8.[a]

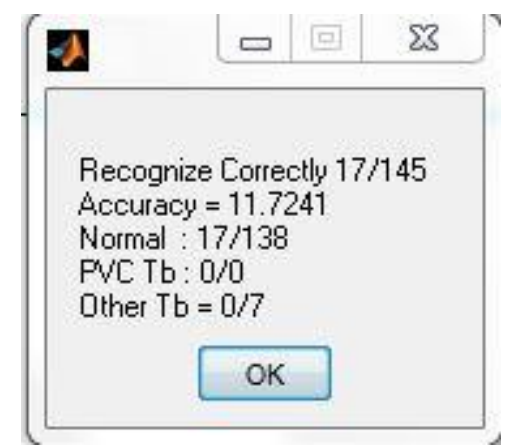

Fig.8.[b]

Fig 8 [a], [b] shows some of the classification results while running MATLAB simulations with the efficiency of approximately $96 \%$.

\section{Conclusion}

Number of different types of techniques are used for arrhythmia classification, ischemia detection, and recognition of chronic myocardial diseases using artificial neural network.

The ANN structured as an autoassociator with the hidden markov model is investigated in cardiovascular disease prediction. ANN approach with wavelet transform as feature extraction are implemented and compared in several previous architectures for cardiovascular disease prediction. The role of different techniques for feature extraction is discussed.

However, it is found that prediction of cardiovascular disease is the complex process with very high level of uncertainty, so ANN is not capable alone to tackle such problems. Hence ANN along with the HMM found out to be efficient solution for the problem with increased convergence rate.

\section{References}

[1] A. Taddei et al., "The European ST-T database: Standard for evaluating systems for the analysis of ST-T changes in ambulatory electrocardiography, "Euro. Heart J., vol. 13, pp. 1164-1172, 1992.

[2] "MIT-BIH database distributor,” Tech. Rep., Beth Israel Hospital, Biomed. Eng., Division KB-26, Boston, MA.

[3] J. J. Bailey et al., "Recommendations for standardization and specification in automated electrocardiography: Bandwidth and digital signal processing," Circulation, vol. 81, 1990.

[4] R. Silipo, A. Taddei, and C. Marchesi, "Continuous monitoring and detection of ST-T changes in ischemic patients," IEEE Comput. Cardiol.,pp. 225-228, 1994.

[5] M. Bianchini, P. Frasconi, and M. Gori, "Learning in multilayered networks used as autoassociators," IEEE Trans. Neural Networks, vol.6, pp. 512-515, 1995

[6] D. E. Rumelhart, G. E. Hinton, and R. J. Williams, "Learning internal representations by error propagation," in Parallel Distributed Processing. Exploration in Microstructure of Cognition, J. A. Feldman et al.,Eds. Cambridge, MA: MIT Press, 1986.

[7] R. Silipo, P. Laguna, C. Marchesi, and R. G. Mark, "Karhunen-Lo`eve transform and artificial neural networks for ST-T analysis," IEEE Comput. Cardiol., pp. 213-216, 1995.

[8] B. A. Pearlmutter, "Learning space trajectories in recurrent neural networks," Neural Comput., vol. 1, no. 2, pp. 263-269, 1989.

[9] P. Frasconi, M. Gori, M. Maggini, and G. Soda, "Unified integration of explicit knowledge and learning by example in recurrent networks,” IEEE Trans. Knowl. Data Eng., vol. 7, pp. 340-346, July 1995.

[10] F. Jager, G. B. Moody, S. Divjak, and R. G. Mark, "Assessing the robustness of algorithms for detecting transient ischemic ST segment changes," in IEEE Comput. Cardiol., pp. 229-232, 1994.

[11] J. L. Willems and E. Lesaffre, "Comparison of multigroup logisitic and linear discriminant ECG and VCG classification," J. Electrocardiol., vol. 20, pp. 83-92, 1987.

[12] J. L. Talmon, Pattern Recognition of the ECG. Berlin, Germany:Akademisch Proefscrift, 1983.

[13] A. Gallin et al., "A computer system for analysis of ST segment changes on 24 hours Holter monitortapes: Comparison with other available systems," J. Amer. Coll. Cardiol., vol. 4, pp. 245-252, 1984. 
[14] A. D. Coast, R. M. Stern, G. G. Cano, and S. A. Briller, "An approach to cardiac arrhythmia analysis using hidden Markov models," IEEE Trans.Biomed. Eng., vol. 37, pp. 826-835, 1990.

[15] L. Edenbrandt, B. Devine, and P. W. Macfarlane, "Neural networks for classification of ECG ST-T segments," J. Electrocardiol., vol. 25, no.3, pp. 167-173, 1992.

[16] T. Stamkopulos, M. Strintzis, C. Pappas, and N. Maglaveras, "One lead ischemia detection using a new back-propagation algorithm and the European ST-T database,” IEEE Comput. Cardiol., pp. 663-666, 1992.

[17] R. Silipo, M. Gori, A. Taddei, M. Varanini, and C. Marchesi, "Classification of arrhythmic events in ambulatory ECG, using artificial neural networks,” Comput. Biomed. Res., vol. 28, pp. 305-318, 1995.

[18] J. H. Frenster, "Neural networks for pattern recognition in medical diagnosis," in Proc. 12th IEEE EMBS Biomed. Eng. Perspectives: Health Care Technol. 1990's Beyond, P. C. Pedersen and B. Onaral, Eds., 1990,pp. 1423-1424.

[19] G. Bortolan and J. L. Willems, "Diagnostic ECG classification based on neural networks," J. Electrocardiol., vol. 26, pp. 75-79, 1994.

[20] A. Taddei et al., "The European ST-T database: Standard for evaluating systems for the analysis of ST-T changes in ambulatory electrocardiography,"Euro. Heart J., vol. 13, pp. 1164-1172, 1992.

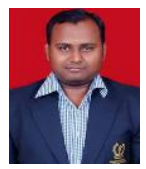

Mr. Ankit S. Sanghavi

Received the B.E. Degree from the Department of Computer Science and Engineering affiliated to Sant Gadge Baba Amravati University, Maharashtra in 2009. He is currently pursuing M.E. in Computer Engineering degree under the guidance of Prof. Sachin M. Bojewar. 